\title{
Advanced Gastric Cancer: Current Treatment Landscape and a Future Outlook for Sequential and Personalized Guide: Swiss Expert Statement Article
}

\author{
Alexander R. Siebenhüner ${ }^{a, b}$ Sara De Dosso ${ }^{c, d}$ Daniel Helbling ${ }^{e}$ \\ Christoforos Astaras $^{f}$ Petr Szturz ${ }^{g}$ Peter Moosmann ${ }^{h}$ Stefanie Pederiva ${ }^{i}$ \\ Thomas Winder ${ }^{\mathrm{e}}$ Philippe Von Burg ${ }^{j}$ Markus Bornerk \\ ${ }^{a}$ Clinic for Medical Oncology and Hematology, University Hospital Zurich and University of Zurich, Zurich, \\ Switzerland; ${ }^{b}$ Cantonal Hospital Schaffhausen, Schaffhausen, Switzerland; ${ }^{\circ}$ Oncology Institute of Southern \\ Switzerland (IOSI), Bellinzona, Switzerland; dUniversità della Svizzera Italiana, Lugano, Switzerland; ${ }^{e}$ OnkoZentrum \\ Zurich, Zurich, Switzerland; fUniversity Hospital of Geneva (HUG), Geneva, Switzerland; ' Lausanne University

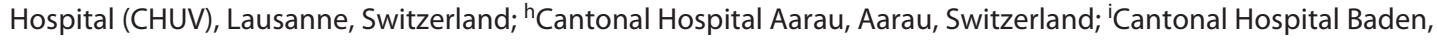 \\ Baden, Switzerland; 'Buergerspital Solothurn, Solothurn, Switzerland; ${ }^{\mathrm{K} O N C O C A R E}$ AG Oncology Centre in the \\ Klinik Engeried, Bern, Switzerland
}

\section{Keywords}

Advanced gastric cancer $\cdot$ Treatment $\cdot$ Trastuzumab ·

Ramucirumab · Nivolumab · Trifluridine-tipiracil

\section{Abstract}

Background: Gastric cancer is a leading cause of cancer-related deaths worldwide. Several treatment possibilities have been investigated, but only a few show clinically meaningful results. Summary: Systemic treatment options for advanced gastric cancer (aGC) have evolved over the recent years, implementing the growing molecular knowledge of this heterogeneous disease. Molecular profiling (at least for HER2-expression, microsatellite instability status, Epstein-Barr virus expression, and programmed death ligand-1 expression/ combined positive score [CPS]) is recommended for all therapy-fit patients prior to the start of a systemic treatment and is crucial for decisions on treatment strategy and drug selection. Various examples like the application of trastuzumab in the HER-2-positive subgroup underline the benefits of this approach starting from the first-line setting. A combination of platinum and fluoropyrimidine remains the first-line chemotherapy backbone in the treatment of advanced gastric cancer. Triplet combinations adding taxanes to the doublet
\end{abstract}

karger@karger.com www.karger.com/ort

Karger ${ }^{\prime \prime}=$ 马OPEN ACCESS
(C) 2021 The Author(s)

Published by S. Karger AG, Basel

This is an Open Access article licensed under the Creative Common Attribution-NonCommercial-4.0 International License (CC BY-NC) (http://www.karger.com/Services/OpenAccessLicense), applicable to the online version of the article only. Usage and distribution for commercial purposes requires written permission. regimen are reserved for certain scenarios. Unfortunately, almost all patients who receive first-line treatment (with or without anti-HER-2 blockade) progress and $<70 \%$ are eligible for a second-line therapy. The addition of monoclonal antibodies has substantially improved outcomes in this setting. As such, ramucirumab has led to significant and clinically meaningful advancements in the second-line treatment. Furthermore, immuno-oncology with checkpoint inhibition and immune stimulation has evolved in the field of aGC. Recent first-line data show a significant survival benefit in aGC patients with a CPS $\geq 5$ under immunochemotherapy. Nonetheless, the impact of immunotherapy combinations and immunochemotherapy remains an area of investigation. Key Message: In this review, we highlight recent improvements in the treatment landscape of advanced gastric cancer, the heterogeneity of this disease, and possible personalized targets.

(C) 2021 The Author(s) Published by S. Karger AG, Basel

\section{Introduction}

Gastric cancer (GC), including adenocarcinoma of the gastroesophageal junction (GEJ) and stomach, is the fifth most common cancer and the third leading cause 
Table 1. TCGA subtypes

\begin{tabular}{|c|c|c|c|c|}
\hline Subtypes & EBV-positive & MSI & GS & CIN \\
\hline Frequency, \% & 8.8 & 21.7 & 19.7 & 49.8 \\
\hline Demographic & Male patients $(81 \%)$ & Old age (median 72 years) & Young age (median 59 years) & No special \\
\hline Histology & & & Diffuse histology & Intestinal histology \\
\hline Main location & Fundus or body $(62 \%)$ & & & GEJ/cardia (65\%) \\
\hline $\begin{array}{l}\text { Molecular } \\
\text { alterations }\end{array}$ & $\begin{array}{l}\text { EBV-CIMP } \\
P D-L 1 / 2, J A K 2 \text { overexpression } \\
\text { Mutation in } P I K 3 C A, A R I D 1 A \text {, } \\
\text { and } B C O R \\
C D K N 2 A \text { silencing } \\
\text { Immune cell signaling } \\
\text { Rare TP53 mutations }\end{array}$ & $\begin{array}{l}\text { Gastric-CIMP } \\
\text { Hypermutation in TP53, } \\
\text { PIK3CA, ERBB3, and ARID1A } \\
\text { MLH1 silencing } \\
\text { Mitotic pathways activation } \\
\text { Commune changes in the genes } \\
\text { of CMHI }\end{array}$ & $\begin{array}{l}\text { CDH1 and RHOA mutation } \\
\text { CLDN18-ARHGAP fusion } \\
\text { Cell adhesion, angiogenesis } \\
\text { pathways enriched } \\
\text { Rare TP53 mutations }\end{array}$ & $\begin{array}{l}\text { TP53 mutation } \\
\text { RTK-RAS activation } \\
\text { Mutations of SMAD4 and } \\
\text { APC }\end{array}$ \\
\hline $\begin{array}{l}\text { Treatment } \\
\text { reaction }\end{array}$ & & $\begin{array}{l}\text { No respond to adjuvant } \\
\text { chemotherapy }\end{array}$ & & \\
\hline Predictive & Yes & Yes & No & No \\
\hline Prognostic & Yes & Yes & Yes & Yes \\
\hline
\end{tabular}

ARID1A, AT-rich interactive domain-containing protein 1A; BCOR, B-cell lymphoma 6 corepressor; CIN, chromosomal instability; GS, genomically stable; GC, gastric cancer; CIMP, CpG island methylator phenotype; DNMT3b, DNA methyltransferase 3b; EBV, Epstein-Barr virus; EGFR, epithelial growth factor receptor; ERBB2, Erb-B2 receptor tyrosine kinase 2; JAK2, Janus-associated kinase 2; LMP2A, latent membrane protein 2A; LELC, lymphoepithelioma-like carcinoma; MSI, microsatellite instability; PI3K, phosphatidylinositol-3-kinase; RHOA, Ras homolog family member A; TCGA, The Cancer Genome Atlas; PD-L1/2, programmed death ligand-1/2; CDK6, cell division protein kinase 6; GEJ, gastroesophageal junction.

of cancer-related death [1]. The incidence of GC is highest in eastern Europe, Eastern Asia, and South America and is greatly dependent on diet and Helicobacter pylori infection [2]. In Western countries, the majority of patients are diagnosed at an advanced stage, which is characterized by inoperable metastatic spread. Although new agents have emerged, long-lasting disease control has not been achieved to this date. Thus, the prognosis of advanced GC remains poor with a 5 -year survival rate of $<10 \%$ [3].

Molecular and histopathological classifications as well as risk factor analyses provide a comprehensive evaluation of gastric adenocarcinoma. On the basis of the Lauren classification, GC is histologically divided into the 2 subtypes: diffuse or intestinal. However, the molecular heterogeneity of GC results in limited utility of traditional morphology-based classification systems, including the World Health Organization (WHO) and Lauren classifications, in guiding clinical treatment [4]. Therefore, patient risk factors must be analyzed in addition to tumor classifications.

The incidence of noncardia GC has decreased due to changes in diet and treatment of chronic Helicobacter pylori infections, accounting for nearly $90 \%$ of new noncardia GC cases [5]. In contrast, GC of the cardia has become more frequent in the Western Hemisphere. Risk factors for GC include obesity, coinfection by Epstein-Barr virus (EBV) or H. pylori and gastroesophageal reflux disease $[6,7]$. Based on these risk factors and the growing knowledge of the tumor microenvironment, subgroups of patients can be identified, making more precise and personalized treatment approaches possible. This is an important step toward the implementation of targeted molecular drugs and immune checkpoint inhibitors in the management of specific patient subgroups.

Environmental and hereditary factors, including germ line alterations of the cadherin-1 gene, result in the development of hereditary diffuse GC [8]. Patients affected by inherited conditions, such as the Lynch syndrome, familial adenomatous polyps, and Peutz-Jeghers syndrome have a substantially higher risk of developing gastric carcinoma [9]. However, classifications based on molecular features are important for risk evaluation in all advanced gastric cancer (aGC) patients. They facilitate the development of sequential systemic treatment pathways for defined subgroups. In this article, we outline the most important aspects regarding molecular marker testing in aGC and recent recommendations concerning related treatments. 


\section{Current Classification Systems and Progress of Molecular Understanding with Clinically Meaningful Impact}

Adenocarcinoma represents the vast majority of GC. Within this group, considerable heterogeneity exists among patients [10]. The traditional morphology-based classification systems include the WHO classification (papillary, tubular, mucinous, signet cell, and poorly cohesive) [11] and the Lauren classification (intestinal, diffuse, and mixed) [12]. A modified WHO classification (differentiated and undifferentiated) has proven helpful in the prediction of the risk of lymph node metastasis in early tumors considered for endoscopic resection, but not in advanced tumors [13]. Given the heterogeneity of GC, molecular analyses were implemented to further improve categorization. This has resulted in several molecular classifications of GC, including intrinsic subtypes, Lei subtypes, the Cancer Genome Atlas (TCGA) subtypes, and Asian Cancer Research Group (ACRG) subtypes, among others [14].

Although the TCGA classification [4] (shown in Table 1) is comprehensive and contains relevant information for clinical use, no classification system comprises all clinically meaningful markers. This would be necessary to optimally guide a personalized approach. Interestingly, the TCGA subtypes differ significantly among Caucasians when compared to the former data, with a lower frequency of microsatellite instability (MSI)-h and EBVpositive aGC $[4,15]$. Many markers can be tested, but only a few are of predictive value, allowing for targeted treatment options. The remaining markers result in various subclassifications and are currently mostly used for prognostication. According to TCGA, GC molecular subtypes include chromosomal instability as the most frequent type represents up to $50 \%$ of the samples, EBVpositive accounting for $9 \%$, MSI-h making up to $21 \%$, and genomically stable accounting for $20 \%$ of the cases.

At the time point of treatment evaluation for aGC, molecular testing of the HER-2 status (expression and amplification) should be performed, initially by immunohistochemistry and then FISH in case of a $2+$ score. For the evaluation of immunotherapy, predictive markers, such as MSI-h/mismatch-repair deficiency (MMR-d) using immunohistochemistry (IHC) or polymerase chain reaction (PCR)-based methods and EBV status should be analyzed using in situ hybridization [16]. Regarding EBV status, the definitive role as a biomarker is not yet fully determined. Evidence exists that EBV-positive GC is potentially associated with immunogenicity and may hence exhibit a greater vulnerability to checkpoint-blockade therapy. To this date, clinical trials have used EBV status as a stratification marker, but not as a definitive biomarker. Therefore, EBV status can be considered a biomarker when evaluating salvage therapy, but not for every aGC patient upfront.

Interestingly, programmed death ligand-1 (PD-L1) status does not only function as a predictive marker for immunotherapy-driven concepts but is also associated with prognosis [17]. However, the prognostic relevance of PD-L1 protein expression in aGC remains controversial and must be interpreted in a differentiated manner $[18,19]$.

This leads directly to the development and use of immunoscores in order to enable a comprehensive evaluation of the tumor as well as environmental factors in terms of immunogenicity. As such, PD-L1 CPS is defined as the ratio of the number of all PD-L1-expressing cells (tumor cells, lymphocytes, and macrophages) to the number of all tumor cells [20]. CPS functions as a predictive marker for checkpoint inhibition and has been used in large clinically relevant trials in aGC. In summary, to select patients for individualized treatment in the firstline setting, we recommend to determine HER-2 status, CPS, and mismatch-repair status.

\section{Clinical Biomarkers with Therapeutic Relevance}

\section{Tyrosine Kinase Inhibition}

Anti-HER-2 Drugs and HER-2 Testing

More than 20\% of GCs show an overexpression and/ or amplification of HER-2. This rate increases to $33 \%$ in GEJ tumors. Importantly, HER-2 testing criteria differ significantly from those routinely applied in breast cancer. Concerning the pattern of reactivity in HER-2-overexpressing cells, the completeness of membrane staining and the number of stained cells necessary to consider a case as positive vary between the 2 entities. Additionally, heterogeneity of HER-2 positivity is more frequent in GC than breast cancer, and a less stringent correlation exists between HER-2 amplification and protein overexpression [17]. In the ToGA trial, adding trastuzumab to chemotherapy showed no benefit in patients with HER-2 amplification and no HER-2 expression (about 20\% of cases) [21]. For this reason, the European Medicines Agency primarily suggests an evaluation of HER-2 status by immunohistochemistry, followed by fluorescence in situ hybridization in case of a $2+$ score [22].

\section{Treatment of HER-2-Positive aGC Patients}

The ToGA trial showed a clear benefit of the addition of trastuzumab to the chemotherapy backbone of cisplatin/5-fluorouracil. Therefore, it is the standard of care in this setting today [18]. Adding trastuzumab to the backbone chemotherapy improved overall survival (OS) significantly from 11.1 months to 13.8 months (HR = 0.74) [21]. Attempts to further improve this standard 
have failed; although the addition of pembrolizumab to cisplatin/5-fluorouracil/trastuzumab seems promising based on phase II trial results [23] and is currently being investigated in a phase III trial (NCT03615626). According to the T-ACT study, continuation of anti-HER-2-directed therapy beyond progression showed no advantage [24]. Moreover, several anti-HER-2 agents, such as pertuzumab (JACOB trial), T-DM1 (GATSBY trial), MM111 (a novel molecule inhibiting heregulin-activated HER-3 signaling in HER-2+ tumors), and tyrosine kinase inhibitors, including lapatinib (LOGIC trial) failed to demonstrate survival benefits in randomized trials in the advanced setting [25-27]. The disappearance of HER-2 positivity after trastuzumab therapy is common [28] and represents the most likely cause of treatment failure in these negative trials. Treatment selection leads to the loss of HER-2 expression and hence promotes the emergence of HER-2 resistance mechanisms [29]. If affected patients progress further, therapy lines follow the recommendations for HER-2-negative aGC. For example, the combination of paclitaxel and ramucirumab represents a second-line option.

In the refractory setting (after 2 or more treatment lines), the randomized phase II DESTINY trial offers a new treatment option for patients who remain clearly HER-2-positive. In this trial, trastuzumab deruxtecan (an antibody-drug-conjugate consisting of an anti-HER-2 antibody and a topoisomerase-I inhibitor) showed a prolongation of the median OS from 8.4 months to 12.5 months $(\mathrm{HR}=0.59, p=0.01)$ when compared to the physician's choice of therapy [30]. FDA approval for this novel treatment option was granted in January 2021. Limiting factors included that the study was exclusively conducted in Japan and Korea.

Further interesting data concerning HER-2-positive GC were presented during ASCO 2020. The phase I/II PANTHERA trial investigated the combination of trastuzumab, pembrolizumab, cisplatin, and capecitabine and met its primary end point, objective response rate (ORR) benefit. With an ORR of 76.7\%, progression-free survival (PFS) of 8.4 months (95\% CI 7.2-22) as well as OS of 18.4 months (95\% CI 17.9-NA), regardless of PD-L1 status, promising observations could be made. This led directly to the phase III trial KN-811 (NCT03615326), which aims to validate the combination in a larger population [31].

Anti-EGFR and Anti-FGFR without Therapeutic

Consequences

Anti-epithelial growth factor receptor (E1GFR) treatment with cetuximab (EXPAND trial) [32] or panitumumab (REAL trial) [33] showed no additional benefit to chemotherapy alone in the first-line treatment of aGC. However, these trials were not biomarker selected for EGFR overexpression. A former retrospective analysis in- dicated that EGFR overexpression might be of predictive value for the susceptibility to anti-EGFR drugs [34]. However, EGFR amplification more likely correlates with the activity of EGFR inhibitors than EGFR overexpression.

Compared with the promising results of fibroblast growth factor receptor-2 (FGFR-2) inhibition in intrahepatic cholangiocellular carcinoma, this target is more complex in GC as FGFR-2 amplification is very heterogeneous within this entity, even in the same patient. This was shown in the SHINE trial using the FGFR-2 inhibitor AZD4547 [35]. Further studies concerning FGFR inhibitors are currently recruiting. During ASCO GI 2021, bemarituzumab, a first-in-class, humanized IgG1 monoclonal antibody that selectively binds to FGFR-2b, showed promising results with a median PFS of 9.4 months compared to 7.4 months in the placebo group. However, the secondary end point was not reached in the phase II FIGHT study [36]. In conclusion, targeting FGFR or members of the EGFR family apart from HER-2 is not of clinical relevance compared to other first-line options in HER-2-negative aGC.

\section{Rare Targets}

To date, no favorable results of MET receptor targeting in aGC have been obtained and neither MET positivity as determined by IHC nor MET gene amplification are of prognostic or predictive value $[37,38]$. BRCA mutations are rare in GC. However, they have clinically relevant implications. These tumor types with a greater amount of DNA damage are sensitive to platinum-based chemotherapy and to poly-ADP-ribose polymerase (PARP) inhibition. Clinical trials investigating PARP maintenance strategies after platinum-based therapyin aGC(NCT03427814) and the use of PARP inhibition in combination with immune checkpoint blockade (NCT02734004) are currently ongoing. The rationale for the combination of PARP inhibition and immune checkpoint blockade is the stimulation of tumor antigen presentation by PARP inhibitors, potentially amplifying the effect of immunotherapy. More DNA damage increases the exposure of tumor antigens, which are targeted by immune-modulating drugs. Furthermore, the combination of PARP inhibitors and antiVEGF antibodies seems promising and is undergoing investigation (NCT03008278).

\section{Early Insights into Possible New Targets}

\section{Anti-Claudin 18.2}

Targeting the tight junction protein claudin seems auspicious. The randomized phase II FAST trial was significantly positive regarding PFS and OS for the combi- 
nation of epirubicin, oxaliplatin, and capecitabine with the first-in-class anti-claudin 18.2 antibody IMAB362 (zolbetuximab, previously known as claudiximab) [39]. However, trials like SPOTLIGHT, investigating the efficacy of zolbetuximab in this setting, are still ongoing (NCT 03504397).

\section{$M M P$}

The inhibition of matrix metalloproteinases with the aim to induce tumor stroma modifications constitutes another therapeutic option currently under investigation. Unfortunately, results of the phase III GAMMA-1 study, presented at the ASCO GI Cancer Symposium 2019, demonstrated no significant difference in outcome for the antibody GS-5745 (andecaliximab) in combination with FOLFOX compared to FOLFOX alone [40].

\section{Era of Immunotherapy and Hints for Predictive Marker Testing}

\section{Mismatch Repair Deficiency}

Several markers are used to predict the efficacy of immunotherapy with immune checkpoint blockade. MSI-h or MMR-d represents the most important and best-established exponent of this group of biomarkers with a reported incidence in GC of $10-22 \%$ in the Western population $[4,41]$. In general, microsatellite instable GC is associated with older age ( $>65$-year-old patients), female gender, onset in the distal stomach, and intestinal histological type according to the Lauren classification. It is more common in patients suffering from multiple synchronous GCs than in those with a solitary tumor [42]. PCR and IHC are the main methods used to detect MSI-h. Molecular testing with PCR allows for a direct detection of MSI-h as a consequence of MMR-d. In the $5-11 \%$ of microsatellite instable malignancies not exhibiting MMR protein loss, usually due to retained antigenicity in an otherwise nonfunctional protein, IHC may underestimate MSI-h cases. In this situation, a PCR-based test is applied to establish the correct diagnosis. Some laboratories use next-generation sequencing (NGS) to determine microsatellite status. In most cases, NGS-based MSI-h analysis requires both tumor and normal tissue. An advantage of this method is the broader range of microsatellite loci included. While the PCR-based method focuses on 5 microsatellite sites, NGS is not limited to these. However, the investment costs per sample are high and the time needed to perform the test is significantly longer than PCR [43].

\section{Epstein-Barr Virus}

According to the literature, approximately $9 \%$ of GCs are EBV-positive [44]. Nonetheless, we observe fewer cases in our clinical experience. Testing is performed using in situ hybridization targeting EBV-encoded small RNA 1 [45]. Although EBV is a strong predictive marker for immunotherapy [46], it is not applied in patient selection for front-line immunotherapy. It is however used as a biomarker in treatment refractory cases. Interestingly, EBV-positive aGC was found to express high levels of PD-L1 in cancer cells and infiltrating immune cells [47]. Moreover, a strong correlation between PD-L1-positive and EBV-positive/MSI-h GC was observed, bringing up the hypothesis that immunotherapy may be as effective in EBV-positive GC patients as it is in MSI-h patients [46]. To this date, some clinical data showing a high efficacy of immunotherapy in EBV-positive aGC could be obtained. However, this concept remains to be proven in clinical trials [46].

\section{Tumor Mutational Burden}

Tumor mutational burden (TMB) is correlated with enhanced clinical response to immunotherapy in melanoma and non-small-cell lung cancer patients [48, 49]. Furthermore, there is evidence that a high TMB could be of predictive value for OS in aGC [48]. As observed in non-small-cell lung cancer, little overlap exists between the TMB-high and PD-L1-positive subgroups [50, 51]. Of note, pembrolizumab has been approved by the FDA for all tumors with a TMB $>10 \mathrm{Mt} / \mathrm{mb}$ based on the results of the $\mathrm{KN}-158$ study.

\section{Programmed Death, Programmed Death Ligand-1,}

and Combined Positive Score

$\mathrm{PD}-\mathrm{L} 1$ expression is commonly used as a predictive marker in aGC, but there are many caveats to be considered. First, PD-L1 does not represent a strong predictive marker for immune checkpoint inhibition and its predictive value varies depending on the immune checkpoint inhibitor used. There are heterogeneous data between responses to nivolumab and pembrolizumab, but this might be more due the different scores used and not of intrinsic different mechanism of both checkpoint inhibitors. In addition, different antibodies are used to measure PD-L1 positivity and poor inter-reader concordance in identifying positive tests is reported in the literature [55]. CPS is an attempt to strengthen the predictive value of PD-L1 and is defined as the number of PD-L1 staining cells (tumor cells, lymphocytes, and macrophages) divided by the total number of viable tumor cells, multiplied by 100 . Concerning nivolumab, PD-L1 positivity measured by CPS, correlates with the efficacy of the substance as shown in the Checkmate-649 trial [52] as well as in a post hoc analysis of Checkmate-032 [53]. Additionally, CPS correlated with avelumab activity in the JAVELIN gastric 100 trial [54]. In summary, data supporting CPS as a predictive marker for the activity of different PD-1/PD-L1 inhibitors in GC are thus becoming more robust. 


\section{Treatment of the Immunogenic aGC}

To date, MSI seems to be the strongest predictive marker for immunotherapy with checkpoint inhibition in GC. In the Keynote-062 trial, an exploratory analysis showed substantial benefit of pembrolizumab monotherapy (arm A) as first-line treatment in patients with MSI tumors. Median OS was not reached for pembrolizumab (95\% CI, 10.7-NR) versus 8.5 months for chemotherapy alone (arm C) (95\% CI, 5.3-20.8), HR = 0.29 [56]. The combination of chemotherapy and pembrolizumab in arm B of the Keynote-062 trial resulted in slightly lower ORR in MSI tumors, favoring pembrolizumab monotherapy. This finding was true for both subgroups of CPS $\geq 1$ and CPS $\geq 10$. The impressive OS difference observed makes pembrolizumab an option for front-line therapy in the MSI patient group. However, the results for the MSI subgroup should be interpreted with caution, taking into account its small size $(n=14)$.

Comparable OS benefits $(\mathrm{HR}=0.33)$ for MSI tumors were observed in the CheckMate-649 trial. Similarly to Keynote-062, this trial was not conducted specifically in a MSI population. The combination of checkpoint blockade and chemotherapy as first-line treatment of aGC was reserved for the CPS $\geq 5$ population (see below) [52]. Consistent with findings regarding upfront treatment, pembrolizumab (vs. paclitaxel) has also demonstrated a clear benefit in MSI patients in the second-line setting (Keynote-061) [57]. In summary, we recommend the earliest possible use of checkpoint inhibitors in the treatment of metastatic MSI gastric cancer.

$\mathrm{PD}-\mathrm{L} 1$ expression/CPS is not as strong of a predictive marker as MSI. Nevertheless, it is widely used in the clinical trial landscape. Recently, the CheckMate-649 trial demonstrated a statistically significant survival benefit with the addition of nivolumab to FOLFOX or CAPOX in the first-line setting of aGC. Clinically meaningful effects were noted especially in the subgroup exhibiting CPS $\geq 5$ [52]. In this population, a median PFS of 7.7 months versus 6.0 months (HR $0.68 ; p<0.0001$ ) and an mOS of 14.4 months versus 11.1 months (HR 0.71; $p<$ 0.0001 ) could be observed. The study had practice changing impacts, leading the FDA to grant priority review to $1 \mathrm{~L}$ nivolumab combinations in gastric/GEJ/esophageal cancers in January 2021.

Currently, checkpoint inhibition plays no role in the second-line treatment of aGC, regardless of PD-L1 expression/CPS $[57,58]$. In the third-line setting, pembrolizumab was approved by the FDA in 2017 (but not EMA or Swissmedic) based on results of the cohort 1 of the Keynote 059 trial. In this single-arm, phase II trial, gastric or GEJ tumors expressing PD-L1 showed a 16\% ORR with responses lasting for 16 months [59]. An alternative checkpoint inhibitor for third-line treatment is nivolum- ab, which is approved for this indication by Swissmedic based on the placebo-controlled ATTRACTION-02 trial [60]. Although nivolumab was active independently of PD-L1 expression, the results of this trial were not widely adopted in Western countries mainly because of the purely Asian study population.

The concept of combinatorial strategies, ranging from immune-immune combinations to immunochemotherapy combinations, is currently under investigation. The phase I/II CheckMate-032 trial showed a higher efficacy of the combination of anti-PD-1 and anti-CTLA-4 than single-agent anti-PD-1 therapy, but higher toxicity levels were observed in the combination group, undermining the clinical relevance of this study [61]. In Keynote-590 [62], the combination of pembrolizumab and a platinum/5FU-based chemotherapy resulted in a clear survival benefit in squamous cell esophageal cancer patients with PD-L1 CPS $\geq 10$ as well as Siewert type 1 GEJ adenocarcinoma patients with PD-L1 CPS $\geq 10$. Median OS of all patients in the intervention group was 13.5 months compared to 9.4 months in the control group (HR 0.64; $p<0.0001$ ). In the squamous cell esophageal cancer subgroup mOS was prolonged from 8.8 months to 13.9 months (HR $0.57 ; p<0.0001$ ). A limiting factor of this study is the fact that regarding adenocarcinomas, only GEJ Sievert type I tumors were included. CM-649, on the other hand, provided data favoring this regimen for first-line treatment of aGC including all adenocarcinoma locations of the stomach. Further combination trials investigating immunochemotherapy (NCT03382600 and NCT03675737) or therapeutic combinations with VEGFR-2 blockade (NCT02999295) and immunotherapy are ongoing.

\section{Treatment of aGC Patients in Absence of Molecular Targets and Immunogenic Profile (HER-2-Negative, Microsatellite Stable), Relevance of Anti-VEGF Targeting}

Although triplet therapy with FLOT has become the new standard in the perioperative setting, we do not recommend the routine use of this regimen as first-line therapy in aGC. The V325 study group reported superiority of docetaxel, cisplatin, and fluorouracil (DCF) in the firstline setting over cisplatin/fluorouracil. In their phase III study, DCF prolonged time to progression ( $32 \%$ risk reduction) as well as OS (21\% risk reduction) [63]. However, triplet therapy also resulted in higher toxicity with a significantly greater amount of grade 3 and 4 side effects, even in the era prior to immunotherapy-chemotherapy combinations. Therefore, we do not see a clear benefit of this triplet chemotherapy in the first-line treatment of aGC. Consistently, a recently published Japanese study 
investigated the addition of a taxane to a cisplatin/S1 backbone. Results showed no survival advantage and toxicity was increased [64]. A smaller randomized AIO phase II trial also failed to demonstrate a PFS benefit of FLOT compared to FLO in patients aged 65 years or older [65]. To conclude, triplet combinations (e.g., DCF or FLOT) should be reserved for carefully selected cases. For example, the phase II FLOT-3 trial [66] tested a valuable concept of surgical removal of primary and metastatic sites after neoadjuvant chemotherapy in highly selected patients with limited metastatic disease with the addition of postoperative FLOT. This study showed favorable survival rates for the included patients, setting up a rationale for future randomized trials.

Furthermore, second-line and consecutive regimens remain a challenge. Evidence regarding checkpoint inhibitor monotherapy and alternative chemotherapeutic approaches like irinotecan is limited. Concerning the second-line use of FOLFIRI, some data are available and can be considered [67]. The RAINBOW trial showed superiority of the combination of ramucirumab/paclitaxel over paclitaxel/placebo after previous platinum-based chemotherapy. The absolute benefit amounted to an OS prolongation of 2.2 months. Ramucirumab/paclitaxel can be considered the standard of care in the second-line setting [68], especially in patients with a good performance status. Other options include monotherapy with ramucirumab, a taxane or irinotecan. Effects of monotherapy are limited in efficacy and duration of response compared to drug combinations. Therefore, this option should be reserved for patients with reduced performance status, whereas drug combinations will not be possible. Moreover, particularly with the upcoming availabilities of firstline combination therapies and second-line immunotherapy options, alternative compounds to VEGF antibodies or mono-agent chemotherapy should be debated (shown in Fig. 1).

In refractory disease, TAS-102 showed a clear survival benefit compared with placebo in the phase III TAGS trial [69]. Since toxicity was manageable and quality of life could be maintained [70], it represents the standard of care in this setting. As mentioned previously, nivolumab is another option demonstrating superiority over placebo in a phase III trial (ATTRACTION-2) [60]. The population of this study comprised exclusively Asian patients, resulting in a rather reluctant use of nivolumab in this setting in the Western world.

\section{Conclusion}

The comprehensive analysis of subgroups, such as defined by the TCGA, adds substantial value to the understanding of GC and provides clinically relevant informa-

\section{Overall Survival: CPS $\geq 5$}

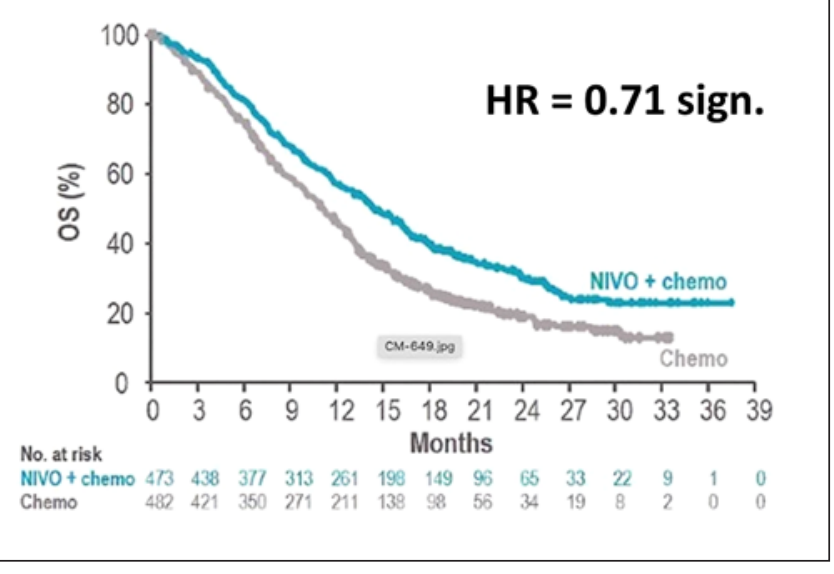

Fig. 1. Second-line therapy: overview. OS, overall survival.

tion. Upfront testing for MSI, HER-2, and PD-L1 CPS is recommended in the aGC setting. If these targets are identified among the tumor characteristics, immune checkpoint blockade or anti-HER-2 antibodies should be added to the treatment regimen according to Checkmate-649 and the ToGa trial, respectively. In case of a reimbursement of the FOLFOX/CAPOX combination with nivolumab, we recommend this therapeutic option as first-line treatment for the PD-L1 CPS $>5$ population. It should be considered as the new standard of care in this subgroup. For the majority of aGC patients, doublet chemotherapy will remain the standard treatment in the first-line setting.

Concerning the second line of therapy, VEGF blockade with or without taxanes is the adequate choice for most patients. However, alternative chemotherapy regimens have been tested and proven to be effective.

Since second-line options have led to an improvement of prognosis, a growing proportion of aGC patients become candidates for third-line therapies. In this situation, TAS-102 and nivolumab have demonstrated significant survival benefits compared with placebo in phase III trials. Nivolumab however is not widely accepted in this setting in the Western world due to an exclusively Asian study population. In some cases, rare targetable mutations are found in aGC, justifying a comprehensive panel analysis early in the treatment history. Hopefully, additional biomarkers will be discovered in the near future, making more personalized treatment choices possible.

To date (March 2021), EMA and Swissmedic approved medications for aGC in the first-, second-, and refractoryline settings comprise platin derivates, $5 \mathrm{FU}$, taxanes, irinotecan, ramucirumab and TAS-102 (only EMA). The approval of pembrolizumab based on the Keynote-590 
trial is pending; Keytruda ${ }^{\circledR}$ monotherapy in MSI-h or TMB-high tumors is neither approved by EMA nor Swissmedic. In order to apply pembrolizumab in this setting, the communication with the insurance company has to be made using the FDA approval statement. EMA approval for nivolumab monotherapy in second- or furtherline therapy was recently withdrawn and, to date, there is no Swissmedic approval for this indication. However, with the expected EMA approval based on the CM-649 trial, the combination of chemotherapy plus nivolumab will be clearly indicated in the first-line treatment of CPSpositive tumors.

\section{Conflict of Interest Statement}

A.R.S has received honoraria due to his scientific consultancy role from AdvancedAcceleratorApp, Amgen, Bayer, BMS, Eisai, Lilly, MSD, Novartis, Pfizer Servier, and Sanofi and has received research grants from Ipsen and Roche. S.D.D. has received consulting honoraria from Amgen, Bayer, BMS, IPSEN, Lilly, Merck, BMS, Novartis, Pfizer, Roche, Sanofi, and Servier, and travel grants from Amgen, BMS, IPSEN, Roche, and Servier. D.H. has received travel grants and congress fees from Servier and Janssen Pharmaceutica and consulting honoraria from Medtalks Switzerland (Play-
ToKnow AG). C.A. has received honoraria from Merck and Servier for participation in Educational Advisory Board-meetings. P.S. has had in the last 3 years or has advisory relationships with: MerckSerono, Servier, and BMS. P.M. has received consultation honoraria from Servier and Bayer, travel support from Bayer. S.P. has received travel grants from Amgen, Celgene, Roche, and Servier. T.W. has nothing to disclose. P.V.B. has received honoraria due to his scientific consultancy role from Astellas, Bayer, BMS, Janssen, MSD, Novartis, Sanofi, and Servier. M.B. has nothing to disclose.

\section{Funding Sources}

This research did not receive any specific grant from funding agencies in the public, commercial, or not-for-profit sector. Medtalks Switzerland (PlayToKnow AG) supported the submission process of this publication.

\section{Author Contributions}

A.R.S. and D.H. designed this review and drafted the initial manuscript. C.A., T.W., S.D.D., P.S., P.M., S.P., and P.V.B. analyzed the data and made substantial contributions to the drafted manuscript. M.B. verified the analytical methods and made final adjustments. All of the authors provided critical feedback, wrote this manuscript, and gave final approval of the submitted version.

\section{References}

1 Ferlay J, Soerjomataram I, Dikshit R, Eser S, Mathers C, Rebelo M, et al. Cancer incidence and mortality worldwide: sources, methods and major patterns in GLOBOCAN 2012. Int J Cancer. 2015 Mar 1;136(5):E359-86.

2 Rawla P, Barsouk A. Epidemiology of gastric cancer: global trends, risk factors and prevention. Prz Gastroenterol. 2019;14(1):26-38.

3 Jim MA, Pinheiro PS, Carreira H, Espey DK, Wiggins CL, Weir HK. Stomach cancer survival in the United States by race and stage (2001-2009): findings from the CONCORD-2 study. Cancer. 2017 Dec 15; 123(Suppl 24):4994-5013.

4 Cancer Genome Atlas Research Network Comprehensive molecular characterization of gastric adenocarcinoma. Nature. 2014 Sep 11;513(7517):202-9.

5 Torre LA, Bray F, Siegel RL, Ferlay J, LortetTieulent J, Jemal A. Global cancer statistics, 2012. CA Cancer J Clin. 2015 Mar;65(2):87108.

6 Akiba S, Koriyama C, Herrera-Goepfert R, Eizuru Y. Epstein-Barr virus associated gastric carcinoma: epidemiological and clinicopathological features. Cancer Sci. 2008 Feb;99(2): 195-201.

7 Bray F, Ferlay J, Soerjomataram I, Siegel RL, Torre LA, Jemal A. Global cancer statistics 2018: GLOBOCAN estimates of incidence and mortality worldwide for 36 cancers in 185 countries. CA Cancer J Clin. 2018 Nov;68(6): 394-424.
8 van der Post RS, Vogelaar IP, Carneiro F, Guilford P, Huntsman D, Hoogerbrugge N, et al. Hereditary diffuse gastric cancer: updated clinical guidelines with an emphasis on germline CDH1 mutation carriers. J Med Genet. 2015 Jun;52(6):361-74.

9 Saigusa S, Tanaka K, Mohri Y, Ohi M, Shimura T, Kitajima T, et al. Clinical significance of RacGAP1 expression at the invasive front of gastric cancer. Gastric Cancer. 2015 Jan;18(1):84-92.

10 Lei Z, Tan IB, Das K, Deng N, Zouridis H, Pattison $\mathrm{S}$, et al. Identification of molecular subtypes of gastric cancer with different responses to PI3-kinase inhibitors and 5-fluorouracil. Gastroenterology. 2013 Sep;145(3):554-65.

11 Bosman FT, Carneiro F, Hruban RH, Theise ND. WHO classification of tumours of the digestive system. 4th ed. 2010.

12 Lauren P. The two histological main types of gastric carcinoma: diffuse and so-called intestinal-type carcinoma. An attempt at a histoclinical classification. Acta Pathol Microbiol Scand. 1965;64:31-49.

13 Gotoda T. Endoscopic resection of early gastric cancer. Gastric Cancer. 2007;10(1):1-11.

14 Wang Q, Liu G, Hu C. Molecular classification of gastric adenocarcinoma. Gastroenterology Res. 2019 Dec;12(6):275-82.

15 Kohlruss M, Grosser B, Krenauer M, SlottaHuspenina J, Jesinghaus M, Blank S, et al. Prognostic implication of molecular subtypes and response to neoadjuvant chemotherapy in 760 gastric carcinomas: role of EpsteinBarr virus infection and high- and low-microsatellite instability. J Pathol Clin Res. 2019 Oct;5(4):227-39.
16 Chen XZ, Chen H, Castro FA, Hu JK, Brenner H. Epstein-Barr virus infection and gastric cancer: a systematic review. Medicine. 2015 May;94(20):e792.

17 Kim JH, Kim SY, Shin EY, Jung JH, Choi HJ, Jun KH. Expression patterns of programmed death-1 and programmed death-1 ligand-1 on T cells in gastric cancer. Oncol Lett. 2019 Sep;18(3):2661-9.

18 Gu L, Chen M, Guo D, Zhu H, Zhang W, Pan J, et al. PD-L1 and gastric cancer prognosis: a systematic review and meta-analysis. PLoS One. 2017;12(8):e0182692.

19 Kim DH, Bae GE, Suh KS, Ryuman D, Song KS, Kim JS, et al. Clinical significance of tumor and immune cell PD-L1 expression in gastric adenocarcinoma. In Vivo. 2020 NovDec;34(6):3171-80.

20 Kulangara K, Zhang N, Corigliano E, Guerrero L, Waldroup S, Jaiswal D, et al. Clinical utility of the combined positive score for programmed death ligand-1 expression and the approval of pembrolizumab for treatment of gastric cancer. Arch Pathol Lab Med. 2019 Mar;143(3):330-7.

21 Bang YJ, Van Cutsem E, Feyereislova A, Chung HC, Shen L, Sawaki A, et al. Trastuzumab in combination with chemotherapy versus chemotherapy alone for treatment of HER2-positive advanced gastric or gastro-oesophageal junction cancer (ToGA): a phase 3, open-label, randomised controlled trial. Lancet. 2010 Aug 28;376(9742):687-97. 
22 Albarello L, Pecciarini L, Doglioni C. HER2 testing in gastric cancer. Adv Anat Pathol. 2011 Jan;18(1):53-9.

23 Janjigian YY, Maron SB, Chatila WK, Millang B, Chavan SS, Alterman C, et al. First-line pembrolizumab and trastuzumab in HER2positive oesophageal, gastric, or gastro-oesophageal junction cancer: an open-label, single-arm, phase 2 trial. Lancet Oncol. 2020 Jun 21(6):821-31.

24 Makiyama A, Sukawa Y, Kashiwada T, Kawada J, Hosokawa A, Horie Y, et al. Randomized, phase II study of trastuzumab beyond progression in patients with HER2-positive advanced gastric or gastroesophageal junction cancer: WJOG7112G (T-ACT study). J Clin Oncol. 2020 Jun 10;38(17):1919-27.

25 Hecht JR, Bang YJ, Qin SK, Chung HC, Xu JM, Park JO, et al. Lapatinib in combination with capecitabine plus oxaliplatin in human epidermal growth factor receptor 2-positive advanced or metastatic gastric, esophageal, or gastroesophageal adenocarcinoma: TRIO013/LOGiC - a randomized phase III trial. J Clin Oncol. 2016 Feb 10;34(5):443-51.

26 Thuss-Patience PC, Shah MA, Ohtsu A, Van Cutsem E, Ajani JA, Castro H, et al. Trastuzumab emtansine versus taxane use for previously treated HER2-positive locally advanced or metastatic gastric or gastro-oesophageal junction adenocarcinoma (GATSBY): an international randomised, open-label, adaptive, phase 2/3 study. Lancet Oncol. 2017 May; 18(5):640-53

27 Tabernero J, Hoff PM, Shen L, Ohtsu A, Shah MA, Cheng K, et al. Pertuzumab plus trastuzumab and chemotherapy for HER2-positive metastatic gastric or gastro-oesophageal junction cancer (JACOB): final analysis of a double-blind, randomised, placebo-controlled phase 3 study. Lancet Oncol. 2018 Oct;19(10): 1372-84.

28 Ishimine $\mathrm{Y}$, Goto A, Watanabe Y, Yajima H, Nakagaki S, Yabana T, et al. Loss of HER2 positivity after trastuzumab in HER2-positive gastric cancer: is change in HER2 status significantly frequent? Case Rep Gastrointest Med. 2015;2015:132030.

29 Aparicio S, Caldas C. The implications of clonal genome evolution for cancer medicine. N Engl J Med. 2013 Feb 28;368(9):842-51.

30 Shitara K, Bang YJ, Iwasa S, Sugimoto N, Ryu $\mathrm{MH}$, Sakai D, et al. Trastuzumab deruxtecan in previously treated HER2-positive gastric cancer. N Engl J Med. 2020 Jun 18;382(25): 2419-30.

31 Rha SY, Lee C-K, Kim HS, Kang B, Jung M, Bae WK, et al. Targeting HER2 in combination with anti-PD-1 and chemotherapy confers a significant tumor shrinkage of gastric cancer: a multi-institutional phase Ib/II trial of first-line triplet regimen (pembrolizumab, trastuzumab, chemotherapy) for HER2-positive advanced gastric cancer (AGC). J Clin Oncol. 2020;38(15_suppl):3081.

32 Lordick F, Kang YK, Chung HC, Salman P Oh SC, Bodoky G, et al. Capecitabine and cisplatin with or without cetuximab for patients with previously untreated advanced gastric cancer (EXPAND): a randomised, open-label phase 3 trial. Lancet Oncol. 2013 May;14(6): 490-9.
33 Waddell T, Chau I, Cunningham D, Gonzalez D, Okines AF, Frances A, et al. Epirubicin, oxaliplatin, and capecitabine with or without panitumumab for patients with previously untreated advanced oesophagogastric cancer (REAL3): a randomised, open-label phase 3 trial. Lancet Oncol. 2013 May;14(6):481-9.

34 Maron SB, Alpert L, Kwak HA, Lomnicki S, Chase L, Xu D, et al. Targeted therapies for targeted populations: anti-EGFR treatment for EGFR-amplified gastroesophageal adenocarcinoma. Cancer Discov. 2018 Jun;8(6): 696-713.

35 Van Cutsem E, Bang YJ, Mansoor W, Petty $\mathrm{RD}$, Chao Y, Cunningham D, et al. A randomized, open-label study of the efficacy and safety of AZD4547 monotherapy versus paclitaxel for the treatment of advanced gastric adenocarcinoma with FGFR2 polysomy or gene amplification. Ann Oncol. 2017 Jun 1;28(6): 1316-24.

36 Wainberg ZA, Enzinger PC, Kang Y-K, Yamaguchi K, Qin S, Lee K-W, et al. Randomized double-blind placebo-controlled phase 2 study of bemarituzumab combined with modified FOLFOX6 (mFOLFOX6) in first-line (1L) treatment of advanced gastric/gastroesophageal junction adenocarcinoma (FIGHT). J Clin Oncol. 2021;39(3_suppl):160.

37 Catenacci DVT, Tebbutt NC, Davidenko I, Murad AM, Al-Batran SE, Ilson DH, et al. Rilotumumab plus epirubicin, cisplatin, and capecitabine as first-line therapy in advanced MET-positive gastric or gastro-oesophageal junction cancer (RILOMET-1): a randomised, double-blind, placebo-controlled, phase 3 trial. Lancet Oncol. 2017 Nov;18(11): 1467-82.

38 Van Cutsem E, Hidalgo M, Canon JL, Macarulla T, Bazin I, Poddubskaya E, et al. Phase I/ II trial of pimasertib plus gemcitabine in patients with metastatic pancreatic cancer. Int J Cancer. 2018 Oct 15;143(8):2053-64.

39 Al-Batran SE, Schuler MH, Zvirbule Z, Zvirbule Z, Manikhas G, Lordick F, et al. FAST: an international, multicenter, randomized, phase II trial of epirubicin, oxaliplatin, and capecitabine (EOX) with or without IMAB362, a first-in-class anti-CLDN18.2 antibody, as first-line therapy in patients with advanced CLDN18.2+ gastric and gastroesophageal junction (GEJ) adenocarcinoma. J Clin Oncol. 2016;34(18_suppl).

40 Shah MA, Yanez Ruiz EP, Bodoky G, Starodub A, Cunningham D, Yip D, et al. A phase III, randomized, double-blind, placebo-controlled study to evaluate the efficacy and safety of andecaliximab combined with mFOLFOX6 as first-line treatment in patients with advanced gastric or gastroesophageal junction adenocarcinoma (GAMMA-1). J Clin Oncol. 2019;37(4_suppl):4.

41 Kim JY, Shin NR, Kim A, Lee HJ, Park WY, Kim JY, et al. Microsatellite instability status in gastric cancer: a reappraisal of its clinical significance and relationship with mucin phenotypes. Korean J Pathol. 2013 Feb;47(1):2835.

42 Nakashima $H$, Honda $M$, Inoue $H$, Shibuta $K$, Arinaga S, Era S, et al. Microsatellite instability in multiple gastric cancers. Int J Cancer. 1995 Aug 22;64(4):239-42.
43 Ratti M, Lampis A, Hahne JC, Passalacqua R, Valeri N. Microsatellite instability in gastric cancer: molecular bases, clinical perspectives, and new treatment approaches. Cell Mol Life Sci. 2018 Nov;75(22):4151-62.

44 Murphy G, Pfeiffer R, Camargo MC, Rabkin CS. Meta-analysis shows that prevalence of Epstein-Barr virus-positive gastric cancer differs based on sex and anatomic location. Gastroenterology. 2009 Sep;137(3):824-33.

45 Nishikawa J, Iizasa H, Yoshiyama H, Shimokuri K, Kobayashi Y, Sasaki S, et al. Clinical importance of Epstein(-)Barr virus-associated gastric cancer. Cancers. 2018 May 29; 10(6):167.

46 Kim ST, Cristescu R, Bass AJ, Kim KM, Odegaard JI, Kim K, et al. Comprehensive molecular characterization of clinical responses to PD-1 inhibition in metastatic gastric cancer. Nat Med. 2018 Sep;24(9):1449-58.

47 Derks S, Liao X, Chiaravalli AM, Xu X, Camargo MC, Solcia E, et al. Abundant PD-L1 expression in Epstein-Barr virus-infected gastric cancers. Oncotarget. 2016 May 31;7(22): 32925-32.

48 Eroglu Z, Zaretsky JM, Hu-Lieskovan S, Kim DW, Algazi A, Johnson DB, et al. High response rate to $\mathrm{PD}-1$ blockade in desmoplastic melanomas. Nature. 2018 Jan 18;553(7688): 347-50.

49 Hellmann MD, Callahan MK, Awad MM, Calvo E, Ascierto PA, Atmaca A, et al. Tumor mutational burden and efficacy of nivolumab monotherapy and in combination with ipilimumab in small-cell lung cancer. Cancer Cell. 2018 May 14;33(5):853-61.e4.

50 Rizvi NA, Hellmann MD, Snyder A, Kvistborg P, Makarov V, Havel JJ, et al. Cancer immunology. Mutational landscape determines sensitivity to PD-1 blockade in non-small cell lung cancer. Science. 2015 Apr 3;348(6230): 124-8.

51 Wang F, Wei XL, Wang FH, Xu N, Shen L, Dai $\mathrm{GH}$, et al. Safety, efficacy and tumor mutational burden as a biomarker of overall survival benefit in chemo-refractory gastric cancer treated with toripalimab, a PD-1 antibody in phase Ib/II clinical trial NCT02915432. Ann Oncol. 2019 Sep 1;30(9):1479-86.

52 Moehler M, Shitara K, Garrido M, Salman P, Shen L, Wyrwicz L, et al. Nivolumab (nivo) plus chemotherapy (chemo) versus chemo as firstline (1L) treatment for advanced gastric cancer/ gastroesophageal junction cancer (GC/GEJC)/ esophageal adenocarcinoma (EAC): first results of the CheckMate 649 study. Ann Oncol. 2020 Sep 1;31(suppl_4):S1191.

53 Lei M, Siemers N, Pandya D, Chang H, Sanchez T, Dorange C, et al. Abstract 2673: association of PD-L1 combined positive score and immune gene signatures with efficacy of nivolumab (NIVO) \pm ipilimumab (IPI) in patients with metastatic gastroesophageal cancer (mGEC). Cancer Res. 2019;79(13 Suppl): 2673.

54 Moehler M, Dvorkin M, Boku N, Özgüroğlu M, Ryu MH, Muntean AS, et al. Phase III trial of avelumab maintenance after first-line induction chemotherapy versus continuation of chemotherapy in patients with gastric cancers: results from JAVELIN gastric 100. J Clin Oncol. 2021 Mar 20;39(9):966-77. 
55 Mehta R, Almhanna K. Predictive markers in gastric cancer immunotherapy treatment-are we there yet? Transl Gastroenterol Hepatol. 2019;4:4.

56 Shitara K, Van Cutsem E, Bang YJ, Fuchs C, Wyrwicz L, Lee KW, et al. Efficacy and safety of pembrolizumab or pembrolizumab plus chemotherapy vs chemotherapy alone for patients with first-line, advanced gastric cancer: the KEYNOTE-062 phase 3 randomized clinical trial. JAMA Oncol. 2020 Oct 1;6(10):1571-80.

57 Shitara K, Özgüroğlu M, Bang YJ, Di Bartolomeo M, Mandalà M, Ryu MH, et al. Pembrolizumab versus paclitaxel for previously treated, advanced gastric or gastro-oesophageal junction cancer (KEYNOTE-061): a randomised, open-label, controlled, phase 3 trial. Lancet. 2018 Jul 14;392(10142):123-33.

58 Bang YJ, Ruiz EY, Van Cutsem E, Lee KW Wyrwicz L, Schenker M, et al. Phase III, randomised trial of avelumab versus physician's choice of chemotherapy as third-line treatment of patients with advanced gastric or gastro-oesophageal junction cancer: primary analysis of JAVELIN Gastric 300. Ann Oncol. 2018 Oct 1;29(10):2052-60.

59 Fuchs CS, Doi T, Jang RW, Muro K, Satoh T, Machado M, et al. Safety and efficacy of pembrolizumab monotherapy in patients with previously treated advanced gastric and gastroesophageal junction cancer: phase 2 clinical KEYNOTE-059 trial. JAMA Oncol. 2018 May 10;4(5):e180013.

60 Kang YK, Boku N, Satoh T, Ryu MH, Chao Y, Kato K, et al. Nivolumab in patients with advanced gastric or gastro-oesophageal junction cancer refractory to, or intolerant of, at least two previous chemotherapy regimens (ONO4538-12, ATTRACTION-2): a randomised, double-blind, placebo-controlled, phase 3 trial. Lancet. 2017 Dec 2;390(10111):2461-71.

61 Janjigian YY, Bendell J, Calvo E, Kim JW, Ascierto PA, Sharma P, et al. CheckMate-032 study: efficacy and safety of nivolumab and nivolumab plus ipilimumab in patients with metastatic esophagogastric cancer. J Clin Oncol. 2018 Oct 1;36(28):2836-44.

62 Kato K, Shah MA, Enzinger P, Bennouna J, Shen L, Adenis A, et al. KEYNOTE-590: phase III study of first-line chemotherapy with or without pembrolizumab for advanced esophageal cancer. Future Oncol. 2019; 15(10):1057-66.

63 Cutsem EV, Moiseyenko VM, Tjulandin S, Majlis A, Constenla M, Boni C, et al. Phase III study of docetaxel and cisplatin plus fluorouracil compared with cisplatin and fluorouracil as first-line therapy for advanced gastric cancer: a report of the V325 study group. J Clin Oncol. 2006;24(31):4991-7.

64 Yamada Y, Boku N, Mizusawa J, Iwasa S, Kadowaki S, Nakayama N, et al. Docetaxel plus cisplatin and S-1 versus cisplatin and S-1 in patients with advanced gastric cancer (JCOG1013): an open-label, phase 3, randomised controlled trial. Lancet Gastroenterol Hepatol. 2019 Jul;4(7):501-10.

65 Al-Batran SE, Pauligk C, Homann N, Hartmann JT, Moehler M, Probst S, et al. The feasibility of triple-drug chemotherapy combination in older adult patients with oesophagogastric cancer: a randomised trial of the
Arbeitsgemeinschaft internistische Onkologie (FLOT65+). Eur J Cancer. 2013 Mar; 49(4):835-42.

66 Al-Batran SE, Homann N, Pauligk C, Illerhaus G, Martens UM, Stoehlmacher J, et al. Effect of neoadjuvant chemotherapy followed by surgical resection on survival in patients with limited metastatic gastric or gastroesophageal junction cancer: the AIO-FLOT3 trial. JAMA Oncol. 2017 Sep 1;3(9):1237-44.

67 Kim JA, Lee J, Han B, Park SH, Park JO, Park YS, et al. Docetaxel/cisplatin followed by FOLFIRI versus the reverse sequence in metastatic gastric cancer. Cancer Chemother Pharmacol. 2011 Jul;68(1):177-84.

68 Wilke H, Muro K, Van Cutsem E, Oh SC, Bodoky G, Shimada Y, et al. Ramucirumab plus paclitaxel versus placebo plus paclitaxel in patients with previously treated advanced gastric or gastro-oesophageal junction adenocarcinoma (RAINBOW): a double-blind, randomised phase 3 trial. Lancet Oncol. 2014 Oct;15(11):1224-35.

69 Shitara K, Doi T, Dvorkin M, Mansoor W, Arkenau H-T, Prokharau A, et al. Trifluridine/tipiracil versus placebo in patients with heavily pretreated metastatic gastric cancer (TAGS): a randomised, double-blind, placebo-controlled, phase 3 trial. Lancet Oncol. 2018 Nov;19(11):1437-48.

70 Tabernero J, Alsina M, Shitara K, Doi T, Dvorkin M, Mansoor W, et al. Health-related quality of life associated with trifluridine/tipiracil in heavily pretreated metastatic gastric cancer: results from TAGS. Gastric Cancer. 2020 Jul;23(4):689-98. 